\title{
Attitude Control of an Axi-Symmetric Rigid Body Using Two Controls without Angular Velocity Measurements Paper
}

\author{
Tawfik El-Sayed Tawfik \\ Department of Mathematics, Faculty of Science, Mansoura University, Mansoura, Egypt \\ Email: tawfikstm@yahoo.com
}

Received March 23, 2013; revised April 23, 2013; accepted May 1, 2013

Copyright (C) 2013 Tawfik El-Sayed Tawfik. This is an open access article distributed under the Creative Commons Attribution License, which permits unrestricted use, distribution, and reproduction in any medium, provided the original work is properly cited.

\begin{abstract}
This paper considers the problem of controlling the rotational motion of an axi-symmetric rigid body using two independent control torques without angular velocity measurements. The control law which stabilizes asymptotically this motion is obtained only in terms of the orientation parameters. Global asymptotic stability is shown by applying LaSalle invariance principal. Numerical simulation is introduced.
\end{abstract}

Keywords: Attitude Control; Rigid Body; Stabilization; Two Controls

\section{Introduction}

A rigid body in general (non-symmetric) is controlled with three independent controls without angular velocity measurements [1-3]. If one of the controls is failure, the rigid body is not controllable. Thus the attitude control of a rigid body motion using two controls is an important control problem.

The angular velocity along the symmetric axis of the rigid body is fixed to its initial value. In this case, two control torques are used to stabilize asymptotically the rotational motion about the symmetric axis. Moreover, the orientation of the symmetric axis is described using stereographic coordinates form direction cosines [4].

Many authors have discussed the attitude control of a rigid body motion using two controls that depend in terms of the angular velocities of the rigid body and the orientation parameters. The stabilization of a zero total angular momentum satellite using two reaction wheels has been shown in $[5,6]$. Two controls which stabilize asymptotically a rigid body motion using matching condition are obtained in terms of the angular velocities of the rigid body [7]. Two controls which stabilize asymptotically an axi-symmetric rigid spacecraft are obtained in terms of the angular velocities of the rigid body and the orientation parameters [8-11]. The angular velocity measurement is noisy. It contains high frequency and random fluctuations. In this paper, two control torques which stabilize asymptotically the rotational motion of an axi-symmetric rigid body are obtained only in terms of the orientation parameters.

The present paper is organized as follows: Section 2 presents dynamic and kinematic equations of an axisymmetric rigid body with two control torques. Section 3 is devoted to obtain the two control torques which stabilize asymptotically the rotational motion of an axi-symmetric rigid body in terms of the orientation parameters. The asymptotic stability of this motion is proved by applying LaSalle invariance principal. Section 4 contains numerical simulation to illustrate the theoretical results of the paper.

\section{Dynamics and Kinematics}

Consider the rotational dynamics of an axi-symmetric rigid body controlled by two independent control torques. Two reference frames are introduced. The first $\hat{n}=\left(\hat{n}_{1}, \hat{n}_{2}, \hat{n}_{3}\right)$ is an inertial reference frame, and the second $\hat{b}=\left(\hat{b}_{1}, \hat{b}_{2}, \hat{b}_{3}\right)$ is a body-fixed reference frame and coincident with the principal axes of inertia of the body. The unit vector $\hat{b}_{3}$ lies along the axis of symmetry. Two control torques $u_{1}$ and $u_{2}$ are applied along the unit vectors $\hat{b}_{1}$ and $\hat{b}_{2}$, respectively (Figure 1). Let $A_{i}$ and $\omega_{i}(i=1,2,3)$ be the principal moments of inertia of the rigid body and the components of the angular velocity of the body referred to the $\hat{b}$ frame, respectively. The dynamic equations take the form: 


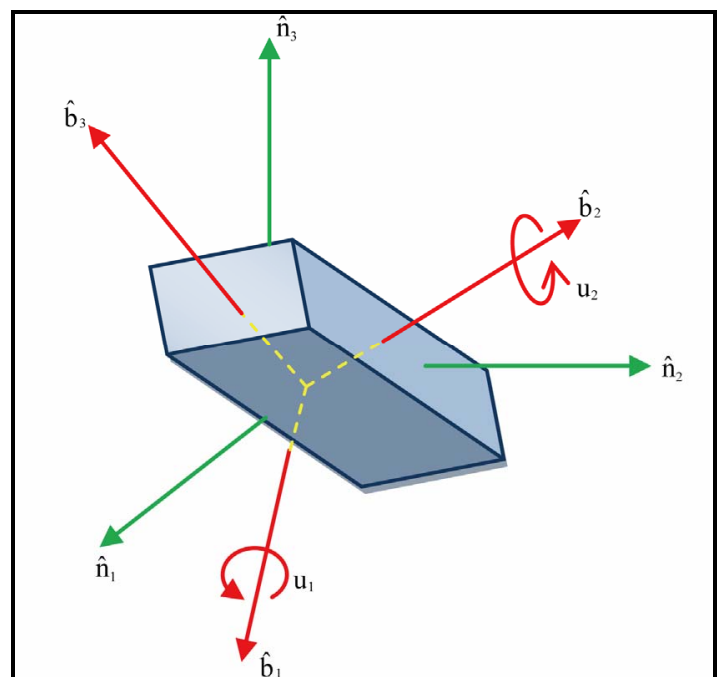

Figure 1. Axi-symmetric rigid body with two controls.

$$
\begin{aligned}
& A_{1} \dot{\omega}_{1}=\left(A_{2}-A_{3}\right) \omega_{2} \omega_{3}+u_{1}, \\
& A_{2} \dot{\omega}_{2}=\left(A_{3}-A_{1}\right) \omega_{3} \omega_{1}+u_{2}, \\
& A_{3} \dot{\omega}_{3}=\left(A_{1}-A_{2}\right) \omega_{1} \omega_{2} .
\end{aligned}
$$

Since $A_{1}=A_{2}=A$, if we let the initial condition $\omega_{3}(0)=\omega_{30}, \omega_{3}$ will remain constant throughout the maneuver. Equations (2.1) can rewrite as:

$$
\begin{aligned}
& A \dot{\omega}_{1}=\left(A-A_{3}\right) \omega_{30} \omega_{2}+u_{1}, \\
& A \dot{\omega}_{2}=\left(A_{3}-A\right) \omega_{30} \omega_{1}+u_{2} .
\end{aligned}
$$

The orientation of an axi-symmetric rigid body is described by using stereographic coordinates form direction cosines [4]. Two orientation parameters $W_{1}$ and $W_{2}$ can be used to describe the position of the $\hat{n}_{3}$ inertial axis in the body fixed $\hat{b}$ frame. These parameters satisfy the differential equation:

$$
\begin{aligned}
& \dot{W}_{1}=\omega_{3} W_{2}+\omega_{2} W_{1} W_{2}+\frac{\omega_{1}}{2}\left(1+W_{1}^{2}-W_{2}^{2}\right), \\
& \dot{W}_{2}=-\omega_{3} W_{1}+\omega_{1} W_{1} W_{2}+\frac{\omega_{2}}{2}\left(1+W_{2}^{2}-W_{1}^{2}\right) .
\end{aligned}
$$

equations (2.2) and (2.3) can be written in a vector form as:

$$
\begin{gathered}
A \dot{\omega}=\left(A-A_{3}\right) S\left(\omega_{30}\right) \omega+u, \\
\dot{W}=S\left(\omega_{30}\right) W+F(W) \omega
\end{gathered}
$$

where

$$
\omega=\left[\begin{array}{ll}
\omega_{1} & \omega_{2}
\end{array}\right]^{\mathrm{T}}, W=\left[\begin{array}{ll}
W_{1} & W_{2}
\end{array}\right]^{\mathrm{T}}, u=\left[\begin{array}{ll}
u_{1} & u_{2}
\end{array}\right]^{\mathrm{T}},
$$

$F(W)$ is the $2 \times 2$ symmetric matrix

$$
F(W)=\frac{1}{2}\left(\left(1-W^{\mathrm{T}} W\right) I+2 W W^{\mathrm{T}}\right)
$$

and $S\left(\omega_{30}\right)$ is the $2 \times 2$ skew-symmetric matrix

$$
S\left(\omega_{30}\right)=\left[\begin{array}{cc}
0 & \omega_{30} \\
-\omega_{30} & 0
\end{array}\right] .
$$

Equations (2.4) and (2.5) can be used to solve the problem of controlling the rotational motion of an axisymmetric rigid body, using Liapunov function technique.

The main objective is to determine the control law $u$ in terms of the orientation parameters that will derive $\omega$ and $W$ to zero. To derive this control law, we introduce the new parameters

$$
\hat{W}=\left[\begin{array}{ll}
\hat{W}_{1} & \hat{W}_{2}
\end{array}\right]^{\mathrm{T}}
$$

which estimate the orientation parameters

$$
W=\left[\begin{array}{ll}
W_{1} & W_{2}
\end{array}\right]^{\mathrm{T}},
$$

respectively. Also we suppose that the orientation parameters and their estimates satisfy the following auxiliary system of differential Equation:

$$
\dot{\hat{W}}=W-\hat{W}+S\left(\omega_{30}\right) W .
$$

Using the kinematic Equation (2.5) the auxiliary system (2.8) can be written in the form:

$$
\dot{\xi}=-\xi+F(W) \omega
$$

where

$$
\xi=W-\hat{W} .
$$

\section{Stabilization Problem}

The main object of this section is to determine the control law $u$ which stabilizes asymptotically the system (2.4), (2.5), (2.9). This control law depends upon the orientation parameters only.

Theorem. The control law

$$
u=-k\left(W^{\mathrm{T}}+\xi^{\mathrm{T}}\right) F(W)
$$

where $k>0$ stabilizes asymptotically the system (2.4), (2.5), (2.9).

Proof. Assume that, the Liapunov function in the form

$$
2 \Phi=A \omega^{\mathrm{T}} \omega+k\left(W^{\mathrm{T}} W+\xi^{\mathrm{T}} \xi\right) .
$$

This function is a positive definite with respect to stabilize variables $\omega, W$, and $\xi$. The time derivative of the Liapunov function (3.2) using (2.4), (2.5), (2.9) and the control law (3.1) takes the form

$$
\begin{aligned}
\frac{\mathrm{d} \Phi}{\mathrm{d} t} & =A \omega^{\mathrm{T}} \dot{\omega}+k\left(W^{\mathrm{T}} \dot{W}+\xi^{\mathrm{T}} \dot{\xi}\right) \\
& =\omega^{\mathrm{T}}\left(u+k\left(W^{\mathrm{T}}+\xi^{\mathrm{T}}\right) F(W)\right)-k \xi^{\mathrm{T}} \xi \\
& =-k \xi^{\mathrm{T}} \xi \leq 0 .
\end{aligned}
$$

The time derivative of the Liapunov function is a nega- 
tive semi-definite function (constant sign function). Thus, under the control law (3.1), the system is stable.

Now, we will prove the asymptotic stability of this system using LaSalle Invariance Principle [12]. Define $\bar{\Omega}$ as the largest invariant set in

$$
\Omega=\{(\omega, W, \xi): \dot{\Phi}=0\}=\{(\omega, W, \xi): \xi=0\} .
$$

On $\bar{\Omega}$ we have that $\dot{\xi}=F(W) \omega=0$ from (2.9). This implies that $\omega=0$ on $\bar{\Omega}$. Since $\bar{\Omega}$ is invariant, $\dot{\omega}=0$ in turn implies

$$
-k W^{\mathrm{T}} F(W)=0
$$

(from (2.4) and (3.1)). This implies that $W=0$ on $\bar{\Omega}$. Therefore

$$
\bar{\Omega}=\{(\omega, W, \xi): \omega=W=\xi=0\} .
$$

\section{Numerical Simulation}

This section shows the effect of the value of the control constant $k$ in control purposes. The Program used in this numerical approach is MAPLE. We choose the inertial moments of an axi-symmetric rigid body, the initial angular velocities of the rigid body, the initial orientation parameters and the initial error attitude parameters as follows:

$$
\begin{aligned}
& A_{1}=A_{2}=15, A_{3}=20 \mathrm{~kg} \cdot \mathrm{m}^{2}, \\
& \omega_{1}(0)=0.2, \omega_{2}(0)=0.2, \omega_{3}(0)=0.1 \mathrm{rad} / \mathrm{s}, \\
& W_{1}(0)=0, W_{2}(0)=0, \\
& \xi_{1}(0)=0, \xi_{2}(0)=0 .
\end{aligned}
$$

Figures 2(a)-(d) show the time response of the body angular velocities, the orientation parameters, the error of the orientation parameters and the control torques, respectively for the control constant $k=2$.

Figures 3(a)-(d) show the time response of the body angular velocities, the orientation parameters, the error of the orientation parameters and the control torques, respectively for the control constant $k=20$.

Figures 4(a)-(d) show the time response of the body angular velocities, the orientation parameters, the error of the orientation parameters and the control torques, respectively for the control constant $k=40$.

Based on the above numerical simulation study we conclude that, increasing the value of the control constant $k$ has the effect of decreasing the convergence behavior of the system. This result is different from the result when the control torques are obtained in terms of the angular velocities of the rigid body and the orientation parameters [11].

\section{Conclusion}

The angular velocity measurement contains high fre-

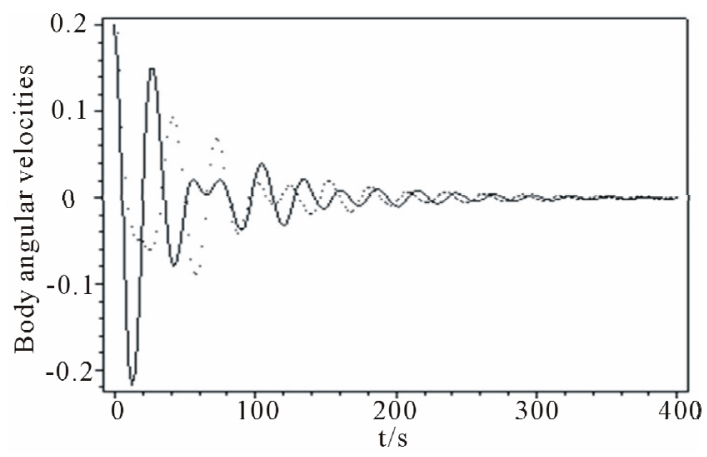

(a)

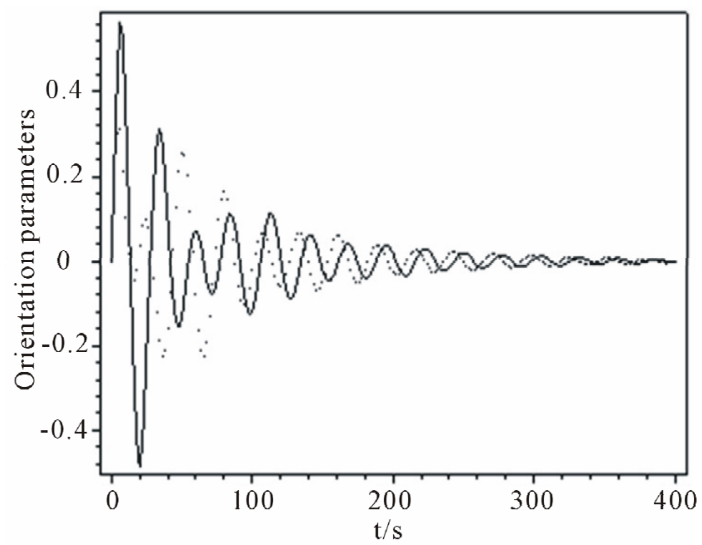

(b)

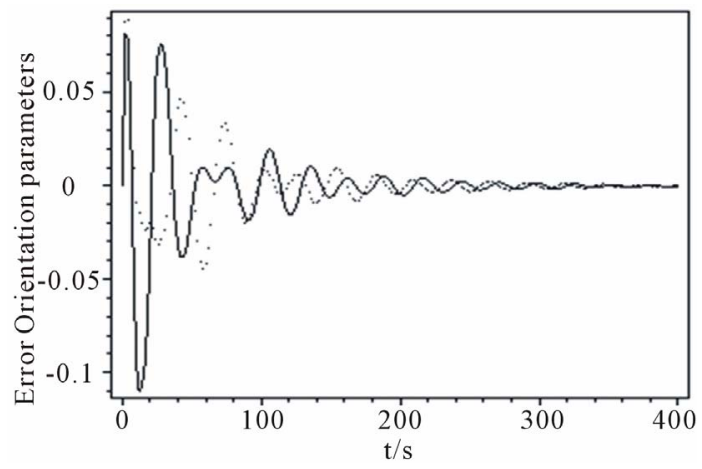

(c)

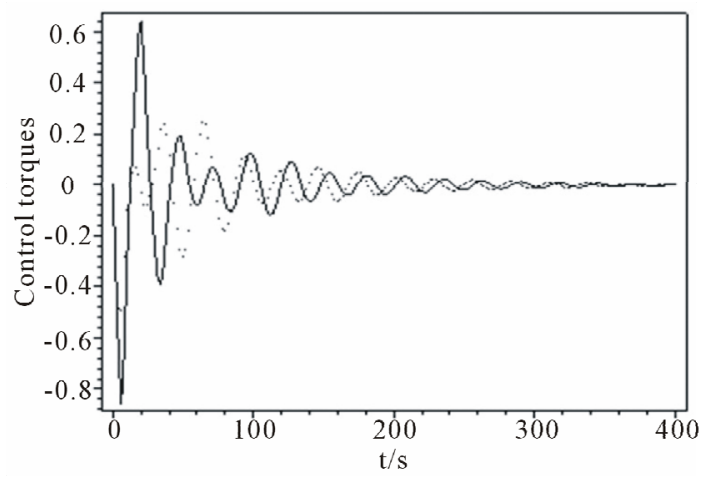

(d)

Figure 2. (a) Body angular velocities; (b) Orientation parameters; (c) Error orientation parameters; (d) Control torques. 


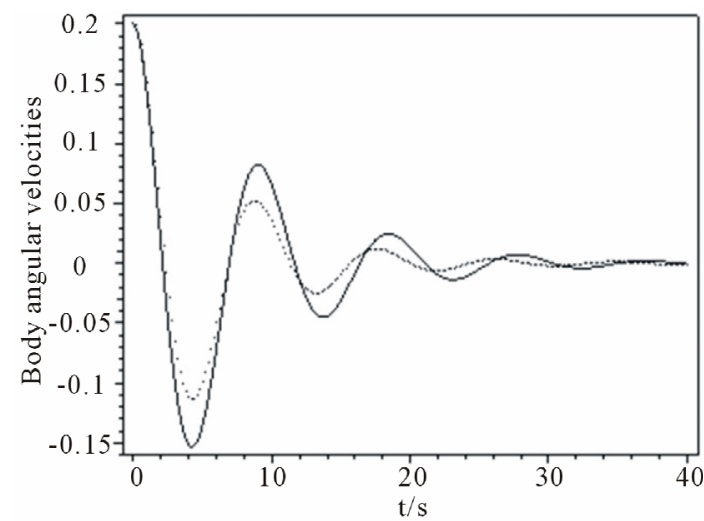

(a)

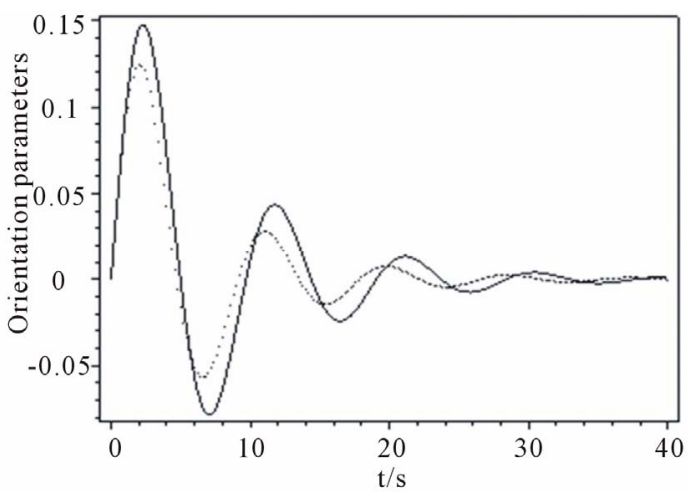

(b)

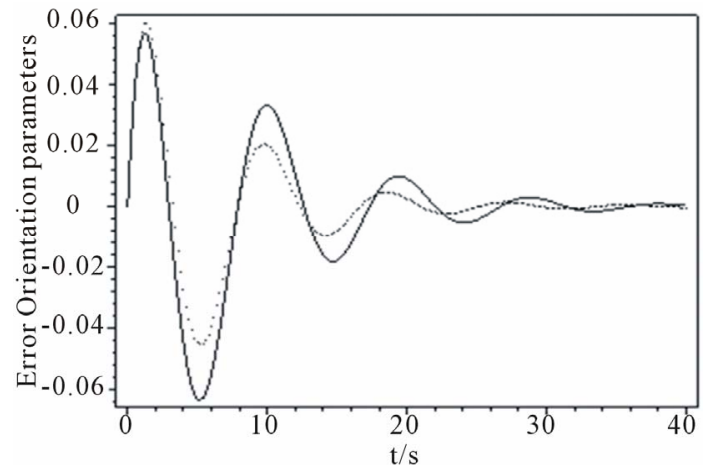

(c)

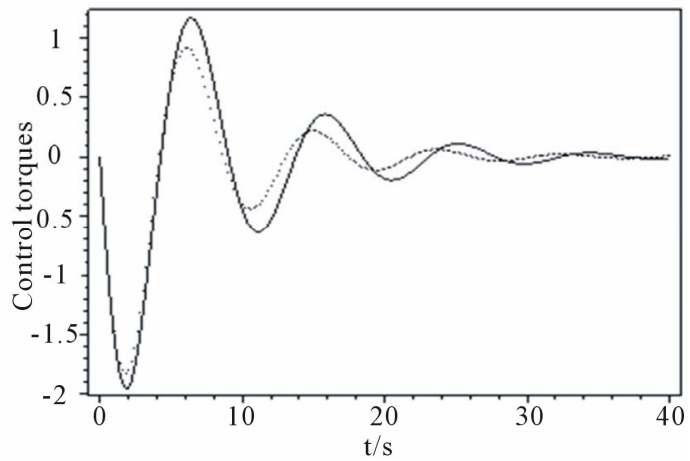

(d)

Figure 3. (a) Body angular velocities; (b) Orientation parameters; (c) Error orientation parameters; (d) Control torques.

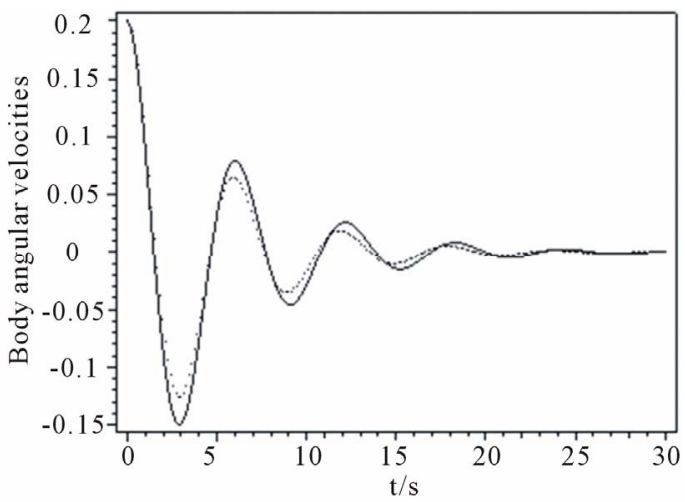

(a)

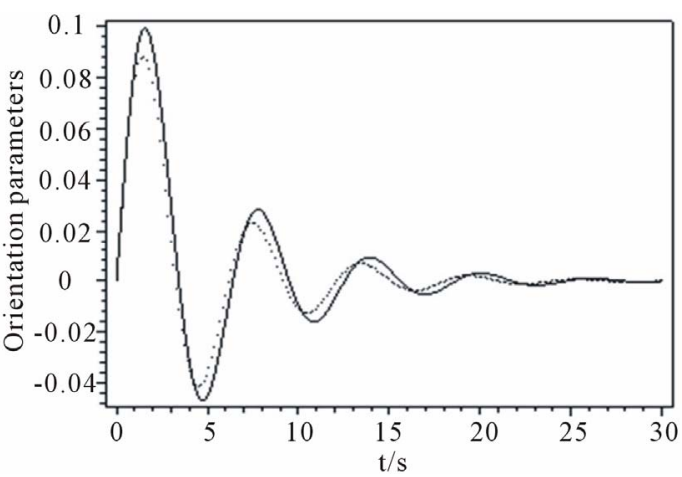

(b)

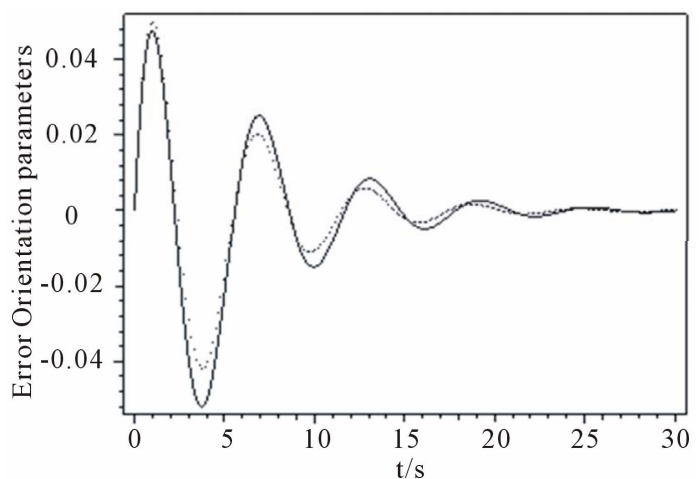

(c)

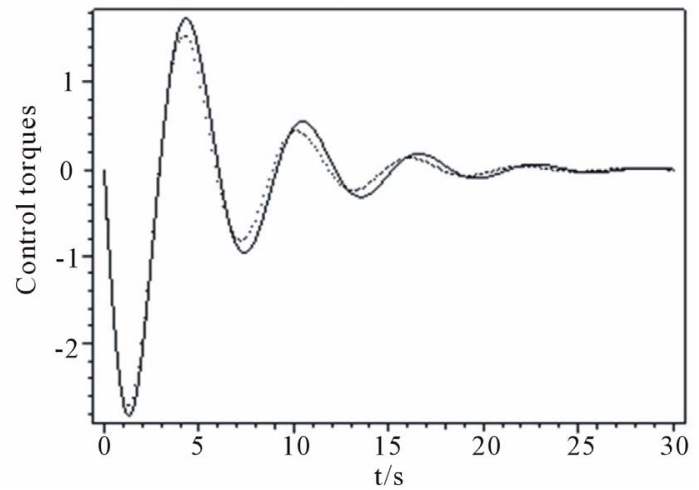

(d)

Figure 4. (a) Body angular velocities; (b) Orientation parameters; (c) Error orientation parameters; (d) Control torques. 
quency and noises or random fluctuations. Two control torques (3.1) which stabilize asymptotically the rotational motion of an axi-symmetric rigid body are obtained in terms of the orientation parameters without angular velocity measurements. Global asymptotic stability is shown by applying LaSalle invariance principal.

\section{REFERENCES}

[1] A. Abdessameud and A. Tayebi, "Global Trajectory Tracking Control of VTOL-UAVs without Linear Velocity Measurements," Automatica, Vol. 46, No. 6, 2010, pp. 1053-1059. doi:10.1016/j.automatica.2010.03.010

[2] S. Ding, S. Li and Q. Li, "Adaptive Set Stabilization of the Attitude of a Rigid Spacecraft without Angular Velocity Measurements," Journal of Systems Science and Complexity, Vol. 24, No. 1, 2011, pp. 105-119. doi:10.1007/s11424-011-8214-1

[3] A. El-Gohary and T. S. Tawfik, "Attitude Stabilization Using Modified Rodrigues Parameters without Angular Velocity Measurements," World Journal of Mechanics, Vol. 1, No. 2, 2011, pp. 57-63. doi:10.4236/wjm.2011.12008

[4] P. Tsiotras and J. M. Longuski, "A New Parameterization on the Attitude Kinematics," Journal of the Astronautical Sciences, Vol. 43, No. 3, 1995, pp. 243-262.

[5] F. Boyer and M. Alamir, "Further Results on the Controllability of a Two-Wheeled Satellite," Journal of Guidance, Control, and Dynamics, Vol. 30, No. 2, 2007, pp.
611-619. doi:10.2514/1.21505

[6] N. M. Horri and S. Hodgart, "Attitude Stabilization of an under Actuated Satellite Using Two Wheels," IEEE Aerospace Conference, Vol. 6, 2003, pp. 2629-2635.

[7] C. Aguilar-Ibaez, M. S. Suarez-Castanon and F. GuzmanAguilar, "Stabilization of the Angular Velocity of a Rigid Body System Using Two Torques: Energy Matching Condition," American Control Conference, Seattle, 11-13 June 2008, pp. 4845-4849.

[8] H. Shen and P. Tsiotras, "Time-Optimal Control of Axisymmetric Rigid Spacecraft Using Two Controls," Journal of Guidance, Control, and Dynamics, Vol. 22, No. 5, 1999, pp. 682-694. doi:10.2514/2.4436

[9] K. Sungpil and K. Youda, "Spin-Axis Stabilization of a Rigid Spacecraft Using Two Reaction Wheels," Journal of Guidance, Control, and Dynamics, Vol. 24, No. 5, 2001, pp. 1046-1049. doi: $10.2514 / 2.4818$

[10] R. Tammepõld, P. Fiorini and M. Kruusmaa, "Attitude Control of Small Hopping Robots for Planetary Exploration: A Feasibility Study," 11th Symposium on Advanced Space Technologies in Robotics and Automation (ASTRA 2011) ESA/ESTEC, Noordwijk, April 2011, pp. 12-14.

[11] P. Tsiotras, "Optimal Regulation and Passivity Results for Axisymmetric Rigid Bodies Using Two Controls," Journal of Guidance, Control, and Dynamics, Vol. 20, No. 3, 1997, pp. 457-463. doi:10.2514/2.4097

[12] H. Khalil, "Nonlinear Systems," MacMillan, Princeton, 1992. 